\title{
Plant Sap Flow Wireless Sensor Network Base on DF-MAC Protocol
}

\author{
Zhang Jiawei ${ }^{1}$, Song Wenlong ${ }^{1}$ and Li Mingbao ${ }^{* 2}$ \\ ${ }^{1}$ College of electromechanical engineering \\ ${ }^{2}$ College of civil engineering \\ Northeast Forestry University \\ Harbin city, P.R. China \\ Zhangjw@nefu.edu.cn
}

\begin{abstract}
Plant sap flow detection plays an important role in water consumption research and plant growth rule. Wireless Sensor Networks (WSN) can effectively realize that transmit the data back to PC. How to prolong the service life of wireless sensor network with the limited energy is a key point. To solve the problem, a novel energy-efficient divisionframe MAC (DF-MAC) protocol applied for Wireless Sensor network to detect plant sap flow is proposed in this paper. The idle listening time is shorten in DF-MAC, and wireless equipment is operated in low power of sleep mode to save energy when data transmission is not occurred. DF-MAC has two major characteristics: low number of collisions and low duty cycle. The nodes of sensor in DF-MAC have shorter listening time that would save the power of communicating with other nodes. The number of collisions is minimized in DF-MAC which can save the energy required to send data packages again. According to the consequences of simulation, the energy depletion of DF-MAC Protocol is much lower compared with the existing MAC. DF-MAC is of theoretical and applied significant for plants sap flow measurement in practice.
\end{abstract}

Keywords: Low energy consumption, DF-MAC protocol, WSN

\section{Introduction}

The research of sap flow is very significant in learning about the water resources consumption research of plant and plant growth rule [1]. In Eco management, if we want to solve the problem of artificial cultivation plants, low survival rate, low preserve rate, low trees growth rate, we should to research the plant sap flow [2]. In the field monitoring process, the wireless network can bring us a lot of convenience when transmitting data or monitoring environment. But for long time detection, how to improve the energy efficiency to prolong the service life of wireless sensor network, become an important problem which we need to solve.

Four main energy waste sources should be considered in WSN [3]: collision result in retransmission, control packet overhead, overhearing, and idle listening. In IEEE 802.11, the energy consumption on idle listening is almost similar with the energy consumption on receiving data, which is nearly $30 \%$ of the total power consumption in WSN [3], so idle listening is the main factor of the WSN energy waste. Some MAC protocoling rules, such as S-MAC [3] and T-MAC [4], adopt the active/sleep cycle plans. Sensor nodes termly switch off their radio and turn into sleep mode that will decrease the idle listening primely. Each frame consists of the active and the sleep time. The duty cycle is the active time divided by the frame duration time. The lower duty cycle is reduced, the more energy can be saved. Many MAC protocols attempt to decrease the duty cycle for higher energy utilization. 
A new MAC protocol plan called division-frame MAC (DF-MAC) protocol is put forward in this paper. This improved algorithm base on S-MAC protocol. In the proposed algorithm the energy depletion is much lower than which in S-MAC and T-MAC by decreasing the number of collisions and decreasing the idle listening time. DF-MAC is a distributed contention-based and self-organizing MAC protocol in which nodes discover their neighbors due to their radio signal level. A central node is not necessary to set to control another nodes.

\section{Related Works}

At present, related researchers have put forward some wireless sensor network MAC protocols with different characteristics. According to the way the access is being controlled, we can classify the MAC protocols into two types: reservation-based and contention-based [5].

MAC protocols are reservation-based could adopt time division multiple access (TDMA), code division multiple access (CDMA) or frequence division multiple access (FDMA), and other ways to achieve no collision between the nodes of the wireless channel allocation. TDMA system meets the needs of saving energy in WSN. But it spends too much to achieve strict time synchronization. Also, the adaptability of TDMA is poor in dynamic topology structure, such as mobile node and node failure. The requirement of time synchronization in FDMA is not as strict as in TDMA. But it cannot avoid collisions completely, especially when network with large size and high density, the network needs enough bandwidth. CDMA enables several nodes communicate in the same time that can increase network throughput, reduce transmission delay. However, the nodes in CDMA need to be coded and decoded complicatedly, so nodes are required to have high computing power. In [6] the author presents a C-MAC protocol is based on BMA protocol for clustered, where the proposed MAC protocol changes the length of frame dynamically in accordance with the number of members of the cluster nodes and communications load in TDMA. C-MAC adapts to these changes of network topology by contending cluster head and slot reassignment. This will cause high energy consumption with heavy network traffic because the work time of cluster-head nodes of this protocol is too long.

The distributed MAC protocol basing on contention adopts the way of applying the channel as needed. Nodes use the wireless channel randomly when nodes need to send or receive data. Network has good expansibility. The most significant things we should consider are the interference between nodes and the consumption of energy caused by collision, idle listening and overhearing. Most distributed contention-based MAC protocol adopts carrier sense multiple access (CSMA) or carrier sense multiple access with collision avoidance (CSMA/CA). In [3] the authors proposed a classical S-MAC protocol. In the active/sleep cycle schemes, the energy consumption in S-MAC is much lower than traditional MAC protocols. The duty cycle in S-MAC is very low. But the network efficiency is reduced. Also, S-MAC can not adapt to the change of network traffic, because of the cycle duration is changeless in S-MAC. When the network traffic is light, the idle listening is increased relatively. This will cause unnecessary energy consumption. In [7] the authors want to increase the energy efficiency as far as possible. When the traffic is disturbed, the proposed protocol will act on the interference node by amplifying the transmitted power of control channel to ensure transmitting the residual data. But the authors ignore that when the node which is high transmitted power after transmitting the current packet, not always is high transmitted power. Because of the proposed protocol adopts "burst" transmission. Also, the transmitted power is minimum transmitted power which is different in each cycle. In [8] the authors improve the 802.11 PSM protocol. It combined with energy saving and power control. But the calculation method of transmission power is not reasonable. In [9] the authors present Sift, a MAC protocol for 
wireless sensor networks that performs well when spatially-correlated contention appears and adapts to changes excellently in the active population size. Unlike other contentionbased MAC protocols, SIFT does not adopt a different contention window, instead, it uses a fixed size contention window. Sift is a simple and easy window-based CSMA protocol which is not complicated to implement. In this protocol, throughput and energy utilization have been improved.

\section{Energy Consumption Analysis}

Fig. 1 shows the overview of the design of DF-MAC. In DF-MAC, time is divided into a number of frames and each frame is divided into two parts: active and sleep. The active cycle is divided into $\mathrm{N}$ sub-frames. Nodes are distributed in the sub-frames where nodes abide by a active/sleep schedule. In varying sub-frames, the schedule is staggered, so the active cycles of the nodes in different sub-frames never overlaps with each other. A node in DF-MAC awakes only at the active cycle of which sub-frames it has been distributed. Which sub-frame the node should be distributed in, there is no restriction. Nodes choose sub-frames randomly. Using sub-frames has three main advantages:

Low energy consumption. Active cycle shortened is proportional to the number of subframes. Therefore, the energy consumption of active cycle in DF-MAC is less than that in S-MAC.

Reduced number of collisions. Nodes are distributed in the different sub-frames. This leads to reduced traffic on average and less probability of collision. Therefore, DF-MAC saves more energy which is used for transmitting the collided packets again.

Longer network lifetime. The more energy we saved, the longer nodes and network lifetime will last.

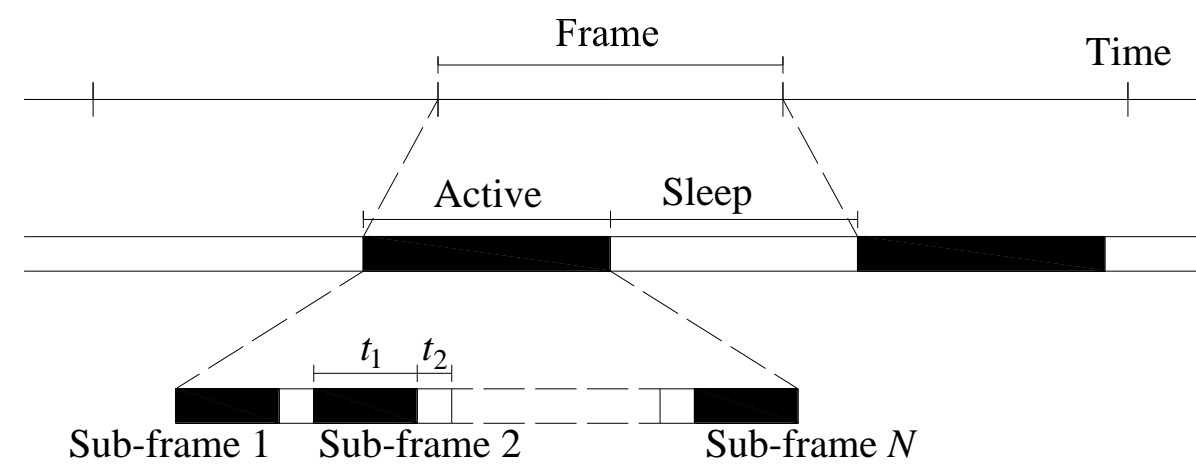

Figure 1. Design Overview of DF-MAC

The shorter listening cycle adds the possibility that a node creates a packet in the sleep mode. This will lead to a longer packet delay. However, delay is not a principal design factor for this system, because the requirement for real time of sap flow detection is undemanding.

This design uses a binary exponential backoff algorithm. The distributed coordination function (DCF), a random access scheme, is based on the CSMA/CA binary exponential backoff algorithm [10-11]. When the node in S-MAC protocol starts to operate, the neighbors should keep synchronized according to the active/sleep mechanism. Node is operated in a fixed length of time. If the scheduling information received on the other nodes during this period, its scheduling cycle is set to be the same as neighbor. Then the SYNC packets broadcast dispatch information after a random period of time that is very small. Including the address of the sending node and the next dormant. If the node receives different scheduling information from neighbor nodes, the first scheduling information will be chosen, then record the scheduling information received by all 
neighbors. If the scheduling information for other nodes did not be received during this period, it will produce its own scheduling cycle and broadcast to the others.

\section{Parameters setting and Simulation}

\subsection{Parameters Setting}

This subsection discusses the numeric area of the design parameters in DF-MAC.

\subsubsection{Frame Duration $T_{\mathrm{f}}$}

$T_{\mathrm{f}}$ is bounded by total active time for all the sub-frames and maximum response time delay $T_{\mathrm{d}}$. We calculate the frame duration by using Eq. 1 .

$$
T_{d} \geq T_{f}>t_{1} \times N
$$

Where $t_{1}$ is the listening cycle for one sub-frame and $N$ is the sub-frames number.

The number of frames $N_{\mathrm{f}}$ is bounded by:

$$
\frac{T_{L}}{T_{d}} \leq N_{f}<\frac{T_{L}}{t_{1} \times N}
$$

Where $T_{\mathrm{L}}$ is the network lifetime.

\subsubsection{Listening Cycle per Sub-frame $t_{1}$}

The battery capacity $C$ and the average node power consumption govern the duration of the listening cycle per sub-frame $t_{1}$ :

$$
t_{1} \leq \frac{C \times V}{\omega \times N_{f}}
$$

Where $V$ is the average output voltage of the battery.

Also, $t_{1}$ is constrained by the time needed to send at least one packet:

$$
t_{1}>d_{S}+d_{t}+2 d_{c}+S d_{S}
$$

Where $d_{S}$ is the propagation delay, $d_{\mathrm{t}}$ is the packet transmission delay, dc is the clock drift delay, and $S$ is the window size.

Thus using Eq. 3 and Eq. $4, t_{1}$ is bounded by:

\subsubsection{Number of Sub-frames $N$}

$$
d_{t}+d_{S}+2 d_{c}+S d_{S}<t_{1} \leq \frac{C \times V}{\omega \times N_{f}}
$$

The average traffic generated per frame in each sub-frame is calculated by using Eq. 6 .

$$
\lambda_{\text {avg }}=n \times \lambda \times T_{f}
$$

The total active time should be greater than the time needed to send the total packet generated by the nodes:

$$
N \times t_{1}>\lambda_{\text {avg }} \times\left(d_{t}+d_{S}+2 d_{c}+\frac{S}{2} d_{S}\right)
$$

The upper limit in $N$ is:

$$
N\left(t_{1}+t_{2}\right) \leq T_{f}
$$

Where $\mathrm{t} 2$ is the guard time between sub-frames. $t_{2}$ is bounded by:

$$
t_{2}>d_{S}+2 d_{c}
$$

Thus using Eq. 7 and Eq. 8, the design bounds of $N$ is: 


$$
\frac{\lambda_{a v g} \times\left(d_{t}+d_{S}+2 d_{c}+(S / 2) d_{S}\right)}{t_{1}}<N \leq \frac{T_{f}}{t_{1}+t_{2}}
$$

In the application, other specifications and requirements can be used to determine the values of these above, such as delay limitations and buffer size in the node. The optimal number of sub-frames should be deployed to get the best performance.

\subsection{Simulation and Results}

We adopt MATLAB to simulate the performance of DF-MAC, and compare the results with other traditional MAC protocols. We make the following assumptions for performing the simulations of DF-MAC:

1. Nodes generate packets that follow Poisson distribution.

2. Time is divided into frames and each frame consists of active and sleep cycle.

3. Each node has three modes of operation: transmit, listen, and sleep.

4. Nodes have unlimited transmit and receive buffer sizes.

5. The wireless channel is no bandwidth constraint.

The parameter values chosen for simulations are summarized in Table 1.

Table 1. Parameter Values Chosen for Numerical Simulations

\begin{tabular}{ll}
\hline Parameter & value \\
\hline Average packet inter-arrival time, $T$ & $2-10[\mathrm{~s}]$ \\
Number of sub-frames, $N$ & $1-10$ \\
Number of nodes, $n$ & 100 \\
Frame duration, $T_{f}$ & $1[\mathrm{~s}]$ \\
Sub-frame duration, $t_{l}$ & $0.3 / N[\mathrm{~s}]$ \\
Window size, $S$ & 8 \\
Node transmitting power & $24.75[\mathrm{~mW}]$ \\
Node listening power & $13.5[\mathrm{~mW}]$ \\
Node sleeping power & $15[\mu \mathrm{W}]$ \\
Node transmission data rate & $19.2[\mathrm{kbps}]$ \\
Average packet length, $\alpha$ & $38[\mathrm{Bytes}]$ \\
Simulation time & $200[\mathrm{~s}]$ \\
\hline
\end{tabular}

\subsubsection{Energy Consumption}

Fig. 2 compares DF-MAC when $\mathrm{N}=3$ with S-MAC. It shows that DF-MAC consumes $62 \%$ less energy than S-MAC when the traffic is heavy, and by $74 \%$ when the traffic is light. The listening cycle per sub-frame in DF-MAC is shorter than S-MAC which results in this reduction of energy consumption. 


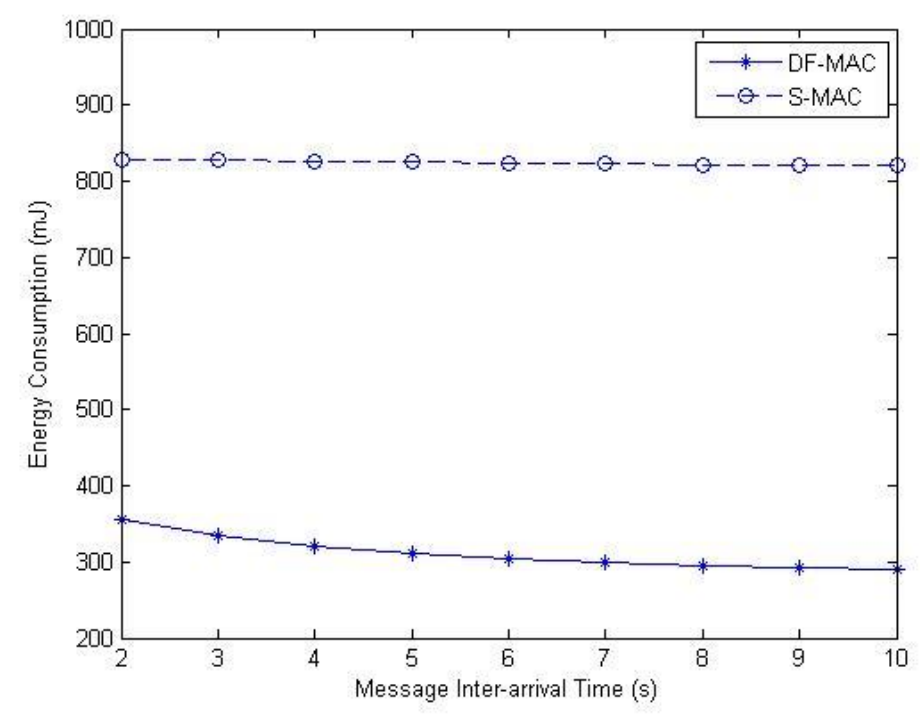

Figure 2. Energy Consumption per node for S-MAC and DF-MAC with $\mathbf{N}=3$

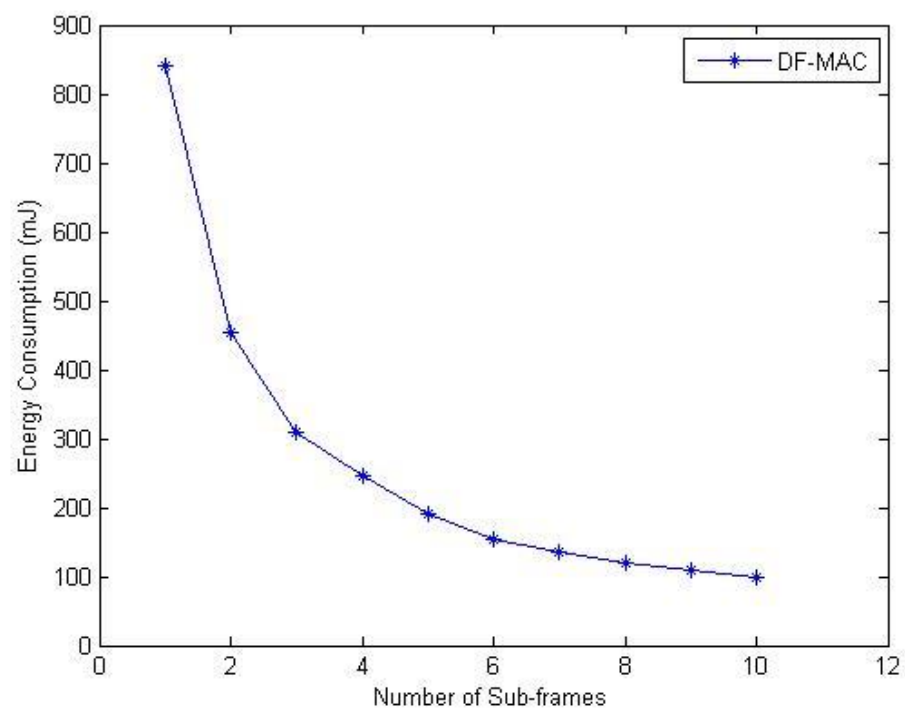

Figure 3. Energy Consumption per node for DF-MAC changes with the Increase in the Number of Sub-frames

The total energy consumed in a node is shown as figure 3 . The number of sub-frames $\mathrm{N}$ is increased from 1 to 10 . The traffic is fixed at 0.2 packet/s.

Energy consumption decreases rapidly up to $\mathrm{N}=6$. However, when the number exceeds 6 , the change of energy consumed is not significant as the nodes spend more time waking up at different schedules and this increases the number of control packets.

\subsubsection{Average Packet Delay}

In DF-MAC the sleeping time is more, thus packets will encounter more delay. The packets delay is composed of two components: queuing delay and transmission delay. Queuing delay is caused by a packet could be destined to another sub-frame or it is stored in the node transmit buffer. 
Therefore, a packet will be delayed in ML-MAC by about one frame. Fig. 4 shows DFMAC has a longer delay than S-MAC.

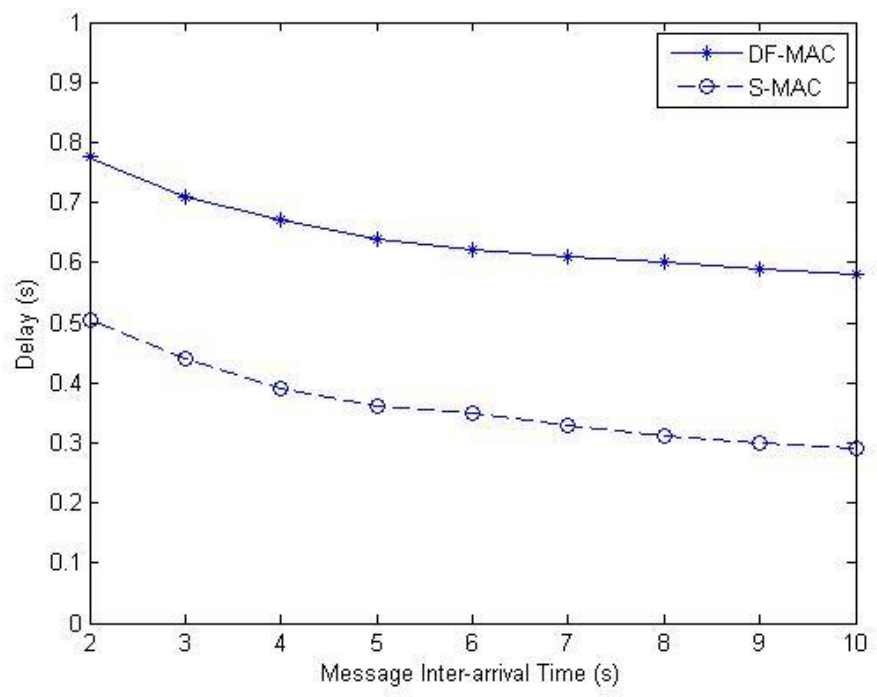

Figure 4. Average Delays for all Packets sent for S-MAC and DF-MAC with N $=3$

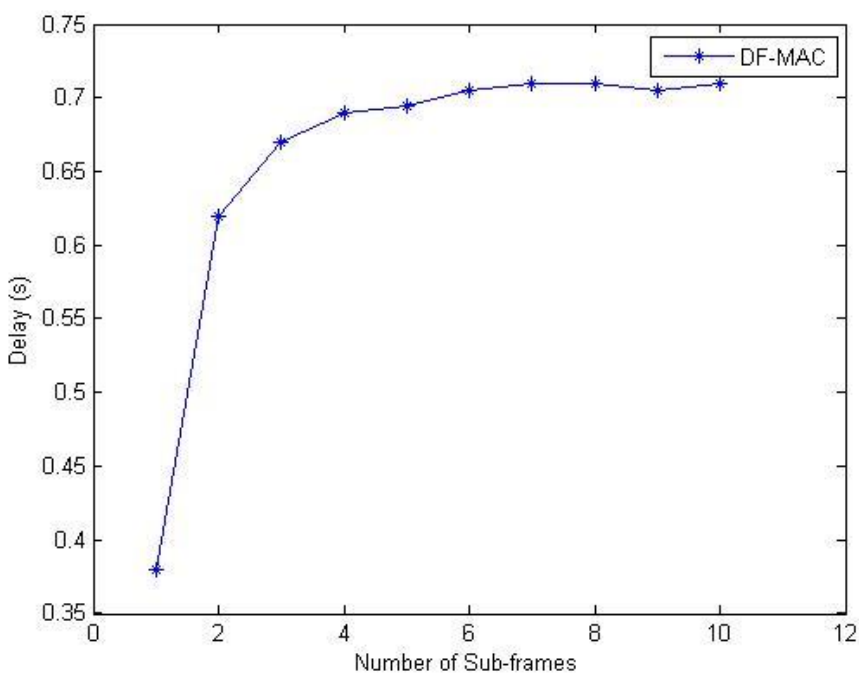

Figure 5. Average Delay for all Packets sent for DF-MAC changes with the Increase in the Number of Sub-frames

Fig. 5 shows the average delay for all packets sent for DF-MAC changes with the increase in the number of sub-frame. The traffic is fixed at 0.2 packet/s. The delay will rapidly increase if the number of sub-frame less than 3 . But, the change of delay is not significant when the number of sub-frame continues to increase because packets are usually buffered for the next or third frame cycle.

\subsubsection{Number of Collisions}

Fig. 7 shows the number of collisions for DF-MAC changes with the increase in the number of sub-frame. The traffic is fixed at 0.2 packet/s. The more number of sub-frames is added, the less number of collisions is. However, when the number exceeds 6 , the 
change of energy consumed is not significant as packet requests have been spread out enough by the sub-frame.

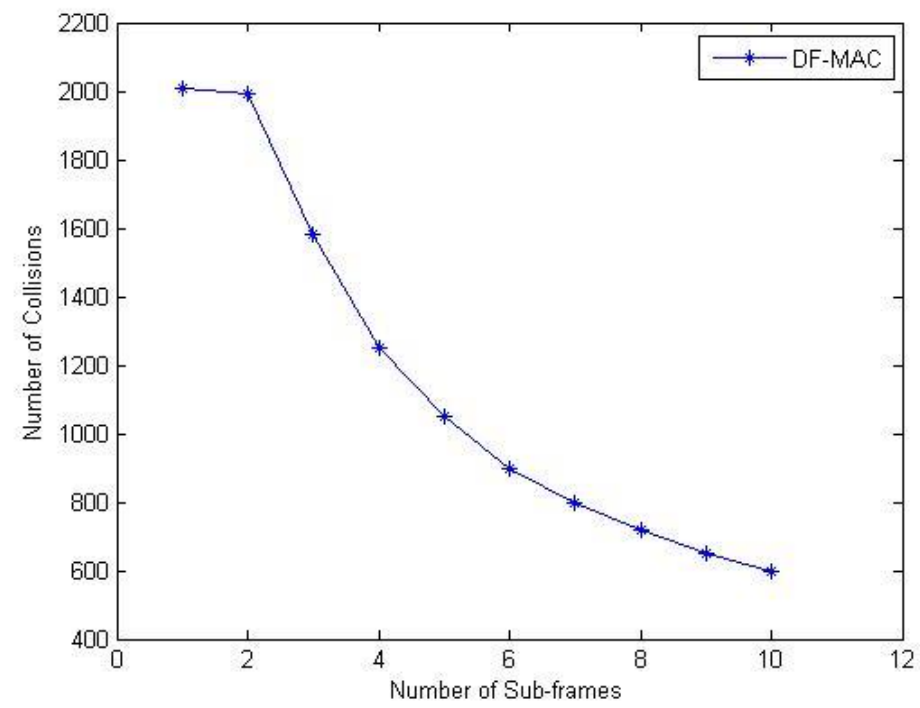

Figure 7. Number of Collisions for DF-MAC changes with the Increase in the Number of Sub-frames

\section{Conclusion}

The research of sap flow plays a significant position in learning about the water consumption research of plant and plant growth rule. As the expansion of the survey area, wireless sensor network become the choice for us to deal with problems. However, when faced with the wild environment, battery is hard to exchange or charge. So energy utilization is the most significant design problem for wireless network. In this paper, an energy-efficient MAC protocol for wireless sensor networks named DF-MAC is proposed. We design a sap flow wireless detection transmission system base on the protocol. In DF-MAC with $\mathrm{N}$ sub-frames, nodes are distributed into $\mathrm{N}$ sub-frames to decrease the idle listening time. The listen cycles of the nodes are non-overlapping in different sub-frames. This will increase energy efficiency from two ways: collision and idle listening. Simulation results show that DF-MAC is superior to S-MAC in power efficiency.

\section{Acknowledgement}

This conclusion described in this paper was supported by a grant from the National Natural Science Foundation of China (31470715) and (31470714), and Fundamental Research Funds for the Central Universities (2572014EB03-02). Professor Li Mingbao as the corresponding author supervised this paperwork is also appreciated.

\section{References}

[1] L. B. William, H. W. Thomas and R. P. Clifford, "A laser diode based system for measuring sap flow by the heat-pulse method. Agricultural and Forest Meteorology", vol. 110, (2002), pp. 275-284.

[2] G. Y. Yue, T. H. Zhang and X. P. Liu, "Development and application of thermal methods in measuring stem sap flow", Scientia Silvae Sincae, vol.42, no. 8, (2006), pp. 102-108.

[3] W. Ye, J. Heideman and D. Estrin, "Medium Access Control with Coordinated Adaptive Sleeping for Wireless Sensor Networks", IEEE/ACM Transactions on Networking, vol. 12, no. 3, (2004), pp. 493506. 
[4] T. V. Dam and K. Langendoen, "Energy-efficient MAC: an adaptive energy-efficient MAC protocol for wireless sensor networks", Proceedings of the jrst international conference on embedded networked sensor systems (SenSys), (2003).

[5] R. Jurdak, C. V. Lopes and P. Baldi, "A survey, classification and comparative analysis of medium access control protocols for ad hoc networks", IEEE Communications Surveys \& Tutorials, (2004), pp. $2-16$.

[6] X. M. Tao, "MAC Protocol for clustered Wireless Sensor Networks", Chinese journal of sensors and actuator, vol. 21, no. 10, (2008), pp. 1785-1788.

[7] L. F. Min, X. W. Jun and G. Chao, "A Power Control MAC Protocol for Wireless Sensor Networks", Journal of Software, vol. 18, no. 5, (2007), pp. 1080-1091.

[8] Z. R. Bo and G. Bo, "Joint power saving and power control MAC protocol in Ad Hoc networks", Journal of Xidian University, vol. 35, no. 3, (2008), pp. 563-571.

[9] K. Jamieson, H. Balakrishnan and Y. Tay, "Sift: a MAC protocol for event-driven wireless sensor networks", Networks and Mobile Systems. Massachusetts Institute of Technology, MIT LCs Publication, MIT-LCS-TR-894, (2003).

[10] G. Bianchi, "Performance Analysis of the IEEE 802.11 Distributed Coordination Function", IEEE Journal on Selected Areas in Communications 2000, (2000), pp. 535-47.

[11] G. B. Yu G. Ding, L. Lin, X. F. Zhao and Y. Zhao, "Aircraft Engine Fuel Flow Prediction Using Process Neural Network", International Journal of Control and Automation, vol. 7, no. 3, (2013), pp. 53-61. 
International Journal of Future Generation Communication and Networking Vol. 9, No.7, (2016) 